\title{
Magnetic energy change available to superconducting condensation in optimally doped $\mathrm{YBa}_{2} \mathrm{Cu}_{3} \mathrm{O}_{6.95}$
}

\author{
HYUNGJE W00 ${ }^{1,2}$, PENGCHENG DAl ${ }^{1,2 *}$, S. M. HAYDEN ${ }^{3}$, H. A. MOOK ${ }^{2 *}$, T. DAHM ${ }^{4}$, D. J. SCALAPIN0 ${ }^{5}$, \\ T. G. PERRING ${ }^{6}$ AND F. DOĞAN7 ${ }^{7}$
}

\author{
${ }^{1}$ Department of Physics and Astronomy, The University of Tennessee, Knoxville, Tennessee 37996-1200, USA \\ ${ }^{2}$ Center for Neutron Scattering, Oak Ridge National Laboratory, Oak Ridge, Tennessee 37831, USA \\ ${ }^{3} \mathrm{H}$. H. Wills Physics Laboratory, University of Bristol, Bristol BS8 1TL, UK \\ ${ }^{4}$ Institut für Theoretische Physik, Universität Tübingen, Auf der Morgenstelle 14, D-72076 Tübingen, Germany \\ ${ }^{5}$ Department of Physics, University of California, Santa Barbara, California 93106, USA \\ ${ }^{6}$ ISIS Facility, Rutherford Appleton Laboratory, Chilton, Didcot, Oxon OX11 0QX, UK \\ ${ }^{7}$ Department of Materials Science and Engineering, University of Missouri-Rolla, Rolla, Missouri 65409-0330, USA \\ *e-mail: daip@ornl.gov; ham@ornl.gov
}

U nderstanding the magnetic excitations in high-temperature (high- $T_{c}$ ) copper-oxide superconductors is important because they may mediate the electron pairing for superconductivity ${ }^{1,2}$. By determining the wavevector $(Q)$ and energy $(\hbar \omega)$ dependence of the magnetic excitations, it is possible to calculate the change in the exchange energy available to the superconducting condensation energy ${ }^{3-5}$. For the high- $T_{c}$ superconductor $\mathrm{YBa}_{2} \mathrm{Cu}_{3} \mathrm{O}_{6+x}$, the most prominent feature in the magnetic excitations is the resonance ${ }^{6-12}$. Suggestions that the resonance contributes a major part of the superconducting condensation $^{4,13}$ have not gained acceptance because the resonance is only a small portion of the total magnetic scattering ${ }^{12-14}$. Here, we report an extensive mapping of magnetic excitations for $\mathrm{YBa}_{2} \mathrm{Cu}_{3} \mathrm{O}_{6.95}\left(T_{\mathrm{c}} \sim 93 \mathrm{~K}\right)$. Absolute intensity measurements of the full spectra allow us to estimate the change in the magnetic exchange energy between the normal and superconducting states, which is about 15 times larger than the superconducting condensation energy ${ }^{15,16}$-more than enough to provide the driving force for high- $T_{\mathrm{c}}$ superconductivity in $\mathrm{YBa}_{2} \mathrm{Cu}_{3} \mathrm{O}_{6.95}$.

If magnetic excitations are mediating electron pairing in the high- $T_{\mathrm{c}}$ copper oxides, it is expected that the change in magnetic exchange energy will provide enough energy for superconducting condensation. The condensation energy is known experimentally from specific heat measurements for $\mathrm{YBa}_{2} \mathrm{Cu}_{3} \mathrm{O}_{6.95}$ (YBCO) to be $\sim 3 \mathrm{~K}$ per formula unit ${ }^{15,16}$ (f.u.). Within the $t-J$ model, the change in magnetic exchange energy can be calculated from the nearest-neighbour spin correlation $s^{3-5}$ between the normal $(N)$ and superconducting $(S)$ states:

$$
\Delta E_{\mathrm{ex}}=2 J\left(\left\langle\mathbf{S}_{i} \cdot \mathbf{S}_{j}\right\rangle_{S}-\left\langle\mathbf{S}_{i} \cdot \mathbf{S}_{j}\right\rangle_{N}\right)
$$

where $J$ is the exchange interaction, and $\boldsymbol{S}_{i}$ and $\mathbf{S}_{j}$ are the electron spin operators at nearest-neighbour $\mathrm{Cu}$ sites $i$ and $j$ in the $\mathrm{CuO}_{2}$ plane, respectively. Instead of estimating the magnetic resonance's contribution to the superconducting condensation ${ }^{4,13}$, here we seek to calculate $\Delta E_{\mathrm{ex}}$ from the entire observable magnetic excitation spectrum. In general, a complete determination of the magnetic excitation spectrum is difficult as spin fluctuations can spread over a large wavevector and energy range. YBCO has two $\mathrm{CuO}_{2}$ planes per unit cell (bilayer) and therefore the magnetic excitations have odd (acoustic) or even (optical) symmetry with respect to the neighbouring planes (Fig. 1). For optimally doped YBCO, the magnetic excitation spectrum is dominated by a resonance mode centred at $41 \mathrm{meV}$ in the acoustic channel ${ }^{6,7}$, and a mapping of the acoustic and optical magnetic excitations should allow an estimation of $\Delta E_{\mathrm{ex}}$.

Figure 1 summarizes the key conclusions of our work. The optical and acoustic spin fluctuations can be separated by their differences in $q_{z}$ dependence (Fig. 1b). The total magnetic response $\chi^{\prime \prime}(Q, \omega)$ can then be written as $\chi^{\prime \prime}\left(q_{x}, q_{y}, q_{z}, \omega\right)=\chi_{\mathrm{a}}^{\prime \prime}\left(q_{x}, q_{y}, \omega\right)$ $\sin ^{2}\left(q_{z} d / 2\right)+\chi_{\mathrm{o}}^{\prime \prime}\left(q_{x}, q_{y}, \omega\right) \cos ^{2}\left(q_{z} d / 2\right)$, where $d=3.342 \AA$ is the spacing between the nearest-neighbour $\mathrm{CuO}_{2}$ planes along the $c$ axis. To probe the entire magnetic spectra in optical and acoustic channels of $\mathrm{YBCO}$, we used the multi-angle position-sensitive spectrometer at the ISIS Facility ${ }^{12,17}$ and chose incident beam energies of $E_{i}=30,40,62.5,75,90,110,130,138,160,210,280,360$ and $450 \mathrm{meV}$ with the incident beam along the $c$ axis. The position-sensitive detectors allow complete determination of the Q-structure of incommensurate spin fluctuations for YBCO in one experimental setting ${ }^{12}$. This avoids the complication of deconvoluting the instrumental resolution necessary for structure determination of incommensurate peaks using triple- 

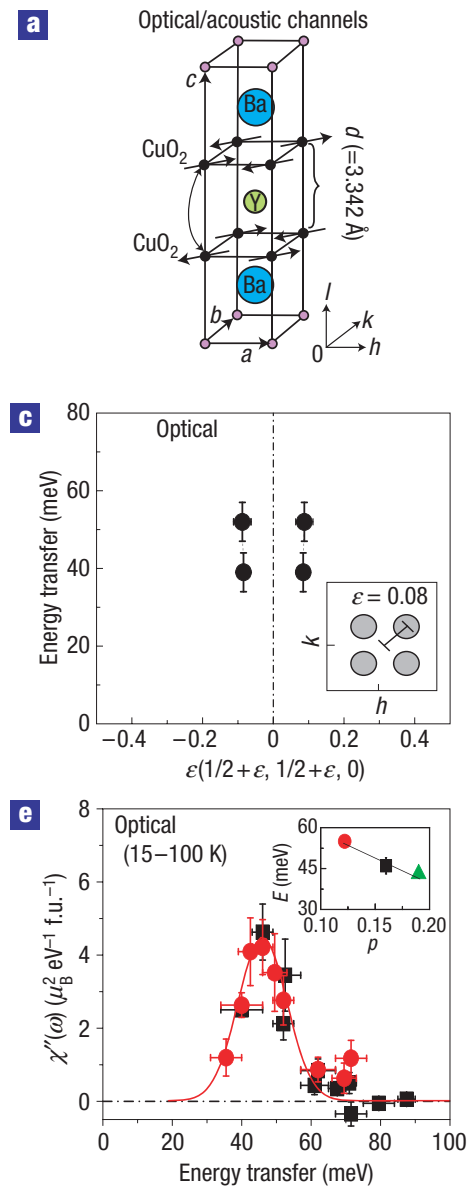
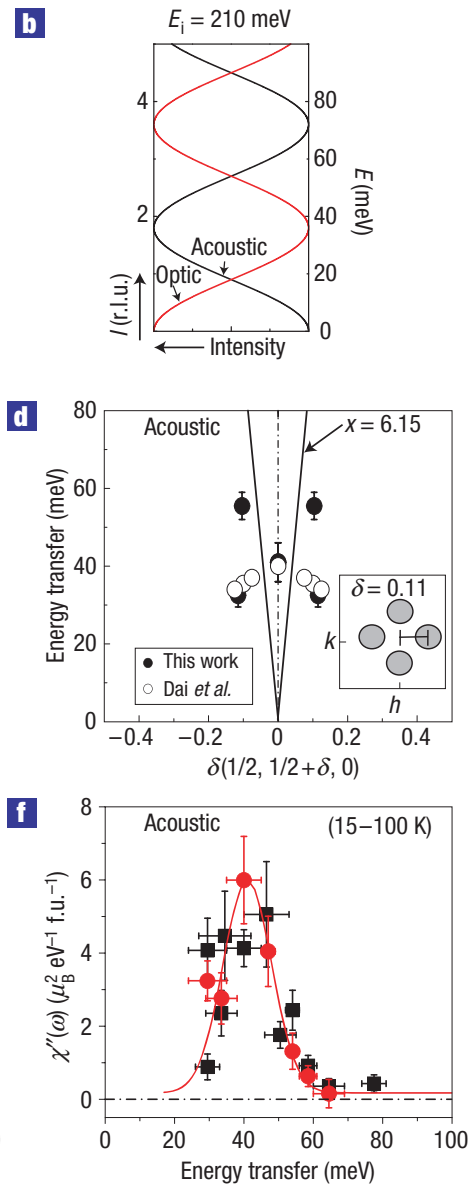

Figure 1 Summary of Q- and $\omega$-dependence of dynamic susceptibility for YBCO. Our experiments were carried out on the $\sim 117 \mathrm{~g} \mathrm{YBCO} \mathrm{single} \mathrm{crystal}\left(T_{\mathrm{c}}=92.5 \mathrm{~K}\right)$ used in previous work ${ }^{10}$. We specify the momentum transfer $\left(q_{h}, q_{k}, q_{l}\right)$ (in units of $\left.\AA^{-1}\right)$ as $(h, k, l)=\left(q_{h} a / 2 \pi, q_{k} b / 2 \pi, q_{l} c / 2 \pi\right)$ in reciprocal lattice units (r.l.u.), where $a=3.82, b=3.88$ and $c=11.68 \AA$ are lattice parameters ${ }^{10}$. $\mathbf{a}$, Schematic diagram for YBCO. $\mathbf{b}$, The $/$ dependence of acoustic and optical spin fluctuations for $E_{i}=210 \mathrm{meV}$. c, Dispersion of constant energy peaks in $\chi_{0}^{\prime \prime}(\mathbf{Q}, \omega)$. The orientation of incommensurate peaks is shown in the inset. $\mathbf{d}$, Dispersion constant energy peaks in $\chi_{\mathrm{a}}^{\prime \prime}(\mathbf{Q}, \omega)$. The solid line shows spin-wave dispersion from undoped $\mathrm{YBa}_{2} \mathrm{Cu}_{3} \mathrm{O}_{6.15}$ (refs 21,22). The inset shows the orientation of the incommensurate spin fluctuations below the resonance energy. e,f, Local susceptibility of 100-15K for optical $\chi_{0}^{\prime \prime}(\omega)$ and acoustic $\chi_{\mathrm{a}}^{\prime \prime}(\omega)$ modes in absolute units. The red circles and black squares are obtained with cuts along the $(h, 1-h)$ and $(h, h)$ directions with an integrating width of \pm 0.15 r.l.u., respectively. The inset in e shows hole-doping dependence of the optical 'resonance' with the red circle and the green triangle from refs 24,25 . The vertical error bars in c,d and the horizontal error bars in e,f indicate the energy integration range. The vertical error bars in $\mathbf{e}$ and $\mathbf{f}$ are statistical uncertainties $(1 \sigma)$.

axis spectroscopy ${ }^{18}$. The temperatures probed were $T=15,100$ and $290 \mathrm{~K}$. The intensity difference between 15 and $100 \mathrm{~K}$ is almost entirely magnetic because of the small value of $Q^{2}$ probed by the experiment and the small change in the Bose factor for $\hbar \omega>30 \mathrm{meV}$ (refs 9,10,19).

Figure $2 \mathrm{a}-\mathrm{c}$ summarizes the temperature dependence of the $34 \leq \hbar \omega \leq 44 \mathrm{meV}$ scattering at the position of optical spin fluctuations $\left(E_{i}=90 \mathrm{meV}\right)$. A clear incommensurate scattering appears at $15 \mathrm{~K}$ (Fig. 2a) and is replaced by a broad response at $100 \mathrm{~K}$ (Fig. 2b). Figure $2 \mathrm{~d}-\mathrm{f}$ shows the temperature dependence of the scattering around the $41 \mathrm{meV}$ acoustic resonance obtained by using $E_{i}=210 \mathrm{meV}$. At $T=15 \mathrm{~K}$, the scattering shows a sharp resonance centred at $(1 / 2,1 / 2)$ (ref. 10). On warming to $100 \mathrm{~K}$, the resonance disappears (Fig. 2e). Further warming to $290 \mathrm{~K}$ does not change the scattering significantly (Fig. 2f).

Figure 3 summarizes the optical spin fluctuations for $31 \leq \hbar \omega \leq 75 \mathrm{meV}$. For $31 \leq \hbar \omega \leq 34 \mathrm{meV}$, the scattering shows no difference between normal and superconducting states (Fig. 3a,e). As there is little normal-state magnetic scattering, there must be an optical spin gap around $34 \mathrm{meV}$. On increasing the energy transfer to $\hbar \omega=39 \pm 5 \mathrm{meV}$, where the acoustic channel has a commensurate resonance, spin fluctuations in the optical channel form a broad incommensurate structure away from $(1 / 2,1 / 2)$ (Fig. 3b). Figure $4 \mathrm{f}$ confirms the incommensurate nature of the scattering and shows that the $(h, h)$ and $(h, 0.5)$ cut directions are non-equivalent. For $\hbar \omega=52 \pm 5 \mathrm{meV}$, again we find incommensurate peaks, but this time the scattering is more box-like with enhanced corners (Fig. $3 \mathrm{c}, \mathrm{g}$ ). The orientation of the scattering is rotated $45^{\circ}$ from that in Fig. $4 \mathrm{~b}$, similar to acoustic high-energy spin excitations in $\mathrm{YBa}_{2} \mathrm{Cu}_{3} \mathrm{O}_{6.6}$ (ref. 12). Finally, on moving the energy to $\hbar \omega=66 \pm 9 \mathrm{meV}$, the temperature difference spectrum is featureless. Inspection of the unsubtracted data at $15 \mathrm{~K}$ reveals no observable magnetic scattering (Fig. $3 \mathrm{~h}$ ).

Figure $4 \mathrm{a}-\mathrm{e}$ shows the intensity difference spectra between $15 \mathrm{~K}$ and $100 \mathrm{~K}$ at various energies in the acoustic channel. For $20 \leq \hbar \omega \leq 27 \mathrm{meV}$, the temperature difference has negative intensity, possibly due to a reduction in the magnetic response on entering the superconducting state $^{19}$ or large phonon population 

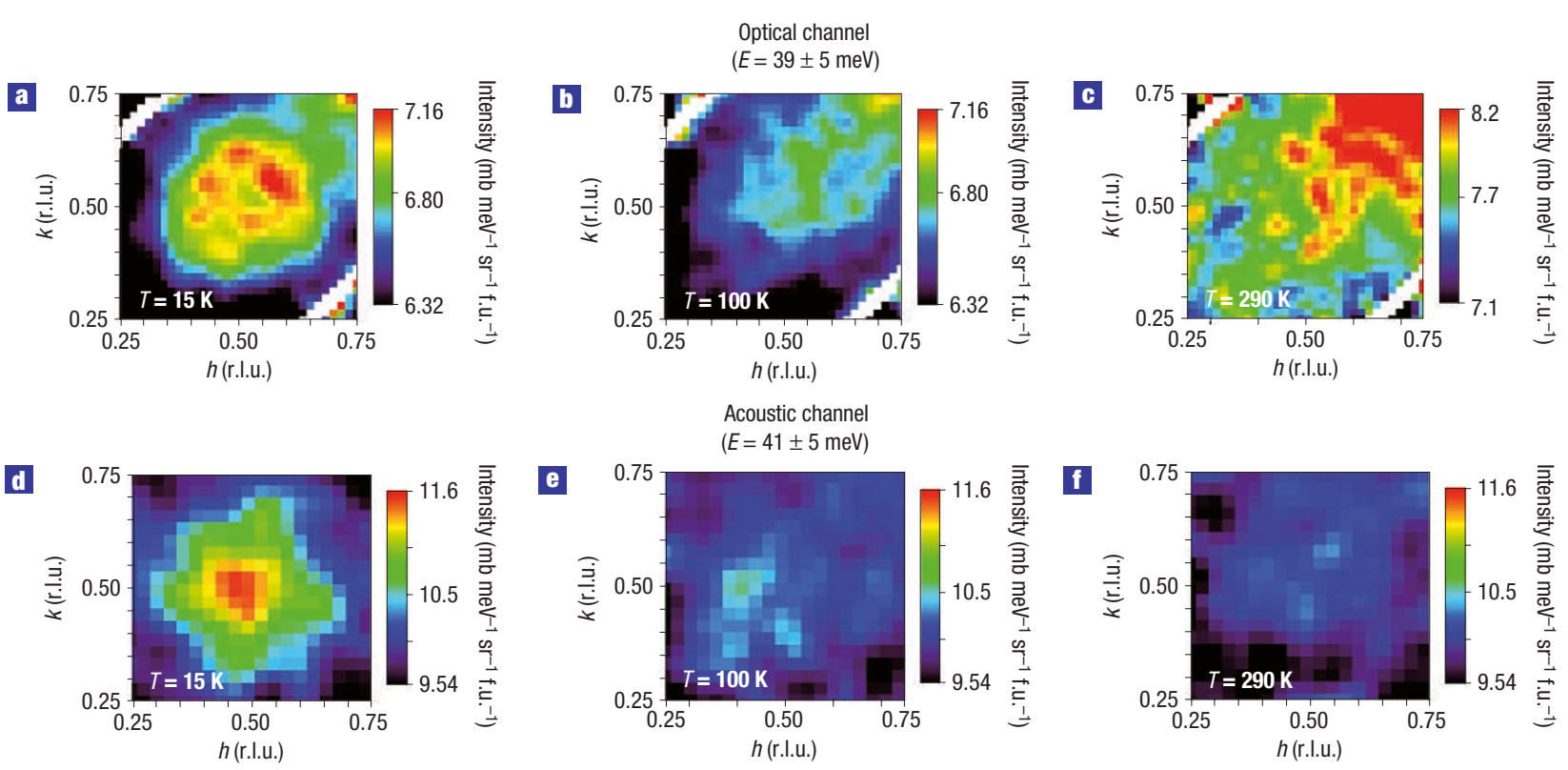

Figure 2 Summary of the temperature dependence of the optical and acoustic scattering at the resonant energy for YBCO. a-f, The temperature dependence of the magnetic scattering around $\hbar \omega \approx 40 \mathrm{meV}$ at optical (a)-(c), $E_{i}=90 \mathrm{meV}$, and acoustic (d)-(f), $E_{i}=210 \mathrm{meV}$ channels. A clear commensurate magnetic scattering is seen at $\sim 40 \mathrm{meV}$ at $15 \mathrm{~K}$ in $\mathbf{d}$, whereas a shows incommensurate scattering. The intensity of phonons increases with increasing temperature and wavevector (b,c).
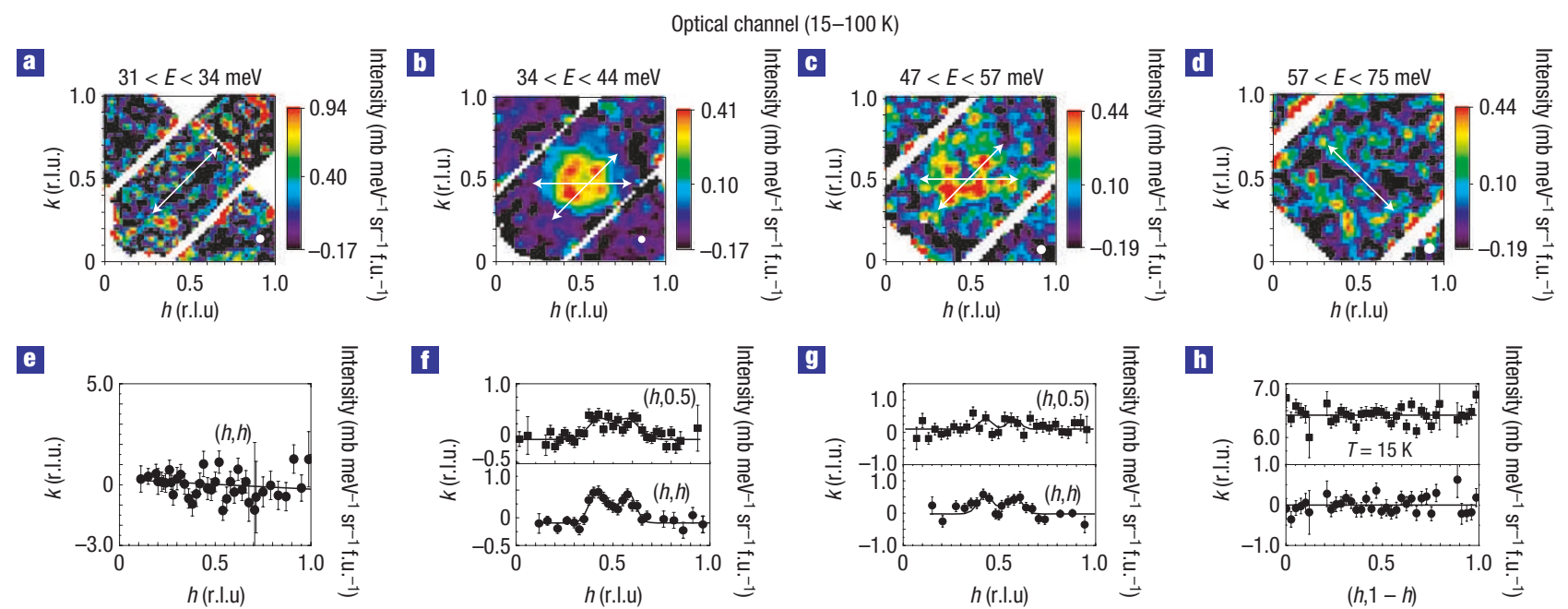

Figure 3 Temperature difference (15-100 K) at various energies for optical mode defined as scattering with $\cos ^{2}\left(q_{z} d / 2\right)>0.8$. a-d, Images obtained with $E_{i}=75,90,130$ and $210 \mathrm{meV}$, respectively. The image at $\hbar \omega=45.5 \pm 1.5 \mathrm{meV}$ does not have enough statistics to determine the $\mathbf{Q}$-structure. e-h, Cuts with $\hbar \omega=32.5 \pm 1.5,39 \pm 5,52 \pm 5$ and $66 \pm 9 \mathrm{meV}$, respectively. The upper panel of $\mathbf{h}$ shows a cut through the unsubtracted data of $\mathbf{h}$ at $15 \mathrm{~K}$. The vertical error bars in $\mathbf{e}-\mathbf{h}$ are statistical uncertainties $(1 \sigma)$.

at $100 \mathrm{~K}$ (Fig. 4a). A cut through the image confirms no intensity gain at low temperatures (Fig. 4f). When increasing the energy to $27 \leq \hbar \omega \leq 35 \mathrm{meV}$, the difference image shows a quartet of incommensurate peaks at $(1 / 2 \pm \delta, 1 / 2)$ and $(1 / 2,1 / 2 \pm \delta)$ with $\delta=0.11 \pm 0.02$ reciprocal lattice units (r.l.u.). To demonstrate that the incommensurate scattering is not circular around $(1 / 2,1 / 2)$, we made two cuts through the image. The cut along the $(h, 1 / 2)$ direction clearly shows two incommensurate peaks around $(1 / 2,1 / 2)$. In contrast, a cut along the $(h, 1-h)$ direction has no incommensurate peaks. Note that a circular symmetry at $\hbar \omega=35 \mathrm{meV}$ has been suggested for YBCO from previous tripleaxis experiments ${ }^{18}$.

Figure $4 \mathrm{c}$ shows the data at the resonance energy and its wavevector dependence has a gaussian lineshape centred around $(1 / 2,1 / 2)$ (Fig. $4 \mathrm{~h})$. For energies above the resonance $(52 \leq \hbar \omega \leq 59 \mathrm{meV})$, the scattering is incommensurate although the low counting rate does not enable an unambiguous identification of its Q-structure (Fig. 4d,i). For $\hbar \omega>60 \mathrm{meV}$, the 

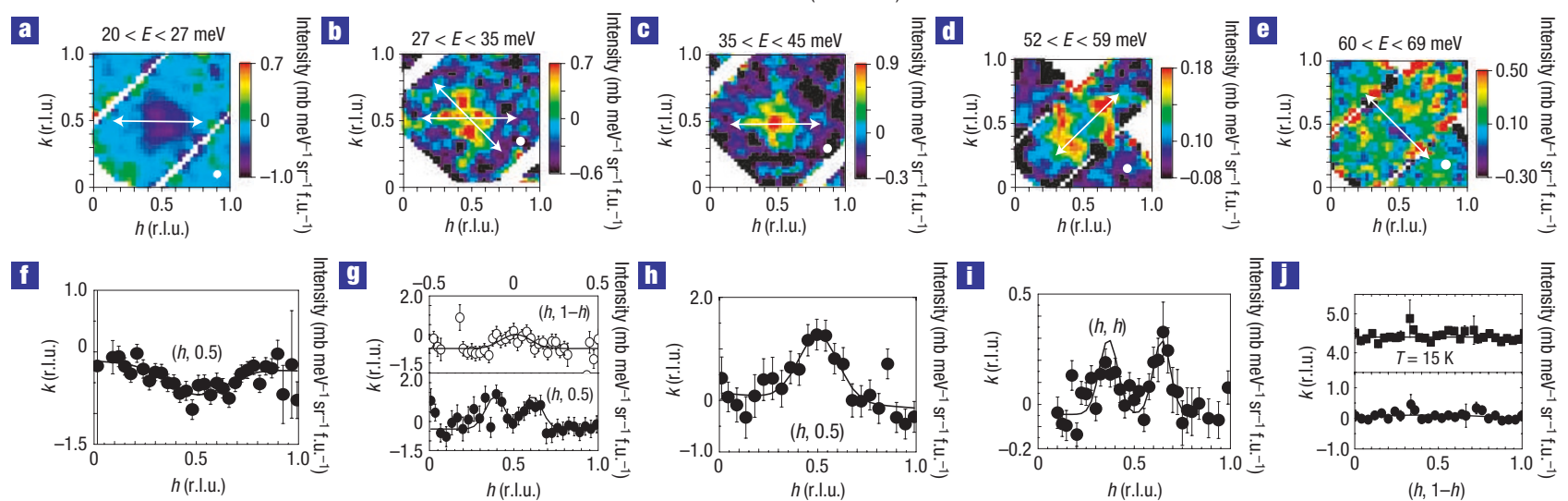

Figure 4 Temperature difference spectra (15-100 K) at various energies for acoustic mode defined as any scattering with $\sin ^{2}\left(q_{z} d / 2\right)>0.8$ (see Fig. 1b). a-e, Images obtained with $E_{i}=90,210,210,90$ and $110 \mathrm{meV}$, respectively. $\mathbf{f}-\mathbf{j}$, Cuts obtained with $\hbar \omega=41 \pm 5,31 \pm 4,40 \pm 5,55.5 \pm 3.5 \mathrm{and} 64.5 \pm 4.5 \mathrm{meV}$, respectively. The upper panel of $\mathbf{j}$ shows a cut through the unsubtracted data of $\mathbf{e}$ at $15 \mathrm{~K}$. The instrumental $Q$-resolutions are marked by white circles in a-e. The vertical error bars in $\mathbf{f}-\mathbf{j}$ are statistical uncertainties $(1 \sigma)$.

temperature difference spectra as well as unsubtracted data show no evidence of magnetic scattering around $(1 / 2,1 / 2)$ (Fig. $4 \mathrm{e}, \mathrm{j})$.

Figure $1 \mathrm{e}$ and $\mathrm{f}$ summarize the superconductivity-induced $(15-100 \mathrm{~K})$ change in the local dynamic susceptibility $\chi^{\prime \prime}(\omega)$ in absolute units for optical and acoustic channels of YBCO, respectively. In the optical channel, $\chi_{0}^{\prime \prime}(\omega)$ has a peak at $\hbar \omega=46$ $\mathrm{meV}$, similar to theoretical expectations ${ }^{20}$. The magnetic spectral weight gradually increases from above the spin-gap value of $\sim 30 \mathrm{meV}$ (ref. 10), peaks at $46 \mathrm{meV}$, and finally diminishes for energies above $\sim 70 \mathrm{meV}$. The acoustic channel behaves similarly although it peaks at the expected resonance position of $40 \mathrm{meV}$ (ref. 10). The magnitude of the total spectral weight in the optical channel $\left\langle m^{2}\right\rangle_{\mathrm{op}}=0.078 \pm 0.02 \mu_{\mathrm{B}}^{2}$ and in the acoustic channel is $\left\langle m^{2}\right\rangle_{\mathrm{ac}}=0.102 \pm 0.02 \mu_{\mathrm{B}}^{2} \mathrm{f}^{\mathrm{u}} \mathrm{.}^{-1}$ respectively. This value is similar to that for $\mathrm{YBa}_{2} \mathrm{Cu}_{3} \mathrm{O}_{6.6}$ around the acoustic resonance energy $\left(\left\langle m^{2}\right\rangle_{\mathrm{ac}}=0.12 \pm 0.02 \mu_{\mathrm{B}}^{2} \mathrm{f}^{\mathrm{u}} \mathrm{.}^{-1} \text { for } 24<\hbar \omega<44 \mathrm{meV}\right)^{12}$. As the high-energy response in underdoped $\mathrm{YBa}_{2} \mathrm{Cu}_{3} \mathrm{O}_{6+\mathrm{x}}(x=0.5,0.6)$ takes up much more spectral weight than the resonance ${ }^{11,12}$, it is surprising that there is essentially no observed magnetic response for energies above $60 \mathrm{meV}$ in YBCO (Fig. 1f). Compared with undoped $\mathrm{YBa}_{2} \mathrm{Cu}_{3} \mathrm{O}_{6.15}$ (refs 21,22), which has a total integrated moment of $\sim 0.4 \mu_{\mathrm{B}}^{2} \mathrm{f}_{\mathrm{u}}{ }^{-1}$ when integrated up to $120 \mathrm{meV}$, the total integrated moment in optimally doped YBCO has only about $26 \%$ of the spectral weight in the same energy range only in the acoustic channel.

Using the spin excitation spectra in Figs 3 and 4, we have calculated the changes in the magnetic excitations from the normal to the superconducting state and estimated $\delta\left\langle\mathbf{S}_{i} \cdot \mathbf{S}_{j}\right\rangle=-0.020 \pm 0.008 \mathrm{f} . u^{-1}{ }^{-1}$, where $\left\langle\mathbf{S}_{i} \cdot \mathbf{S}_{j}\right\rangle$ is the spin-spin correlation function for nearest-neighbour copper atoms (see Supplementary Information). This estimate neglects contributions from energies below $24 \mathrm{meV}$ and above $70 \mathrm{meV}$, where magnetic scattering is difficult to resolve. Also, in equation (1) the difference between the normal and superconducting states is meant to be determined at the same temperature, whereas here we had to take normal-state data at $100 \mathrm{~K}$ and superconducting-state data at $15 \mathrm{~K}$, neglecting a possible temperature dependence of the normal-state magnetic excitations. To assess the error introduced by these neglections, we have fitted an RPA-BCS (random phase approximation-Bardeen-Cooper-Schrieffer) model calculation of the spin excitation spectrum ${ }^{23}$ to our data and calculated the missing contributions within this model. This calculation indicates that our value for $\Delta E_{\mathrm{ex}}$ could be of the order $30 \%$ too large due to these neglections (see Supplementary Information).

Assuming an exchange coupling of $J=100 \mathrm{meV}$, the change in exchange energy would be $\Delta E_{\mathrm{ex}}=2 J \delta\left\langle\mathbf{S}_{i} \cdot \mathbf{S}_{j}\right\rangle=-4.1 \mathrm{meV} \mathrm{f.u} .^{-1}=$ $-24 \mathrm{~K}$ per planar $\mathrm{Cu}$. This value is a factor of 1.3 times larger than the $18 \mathrm{~K}$ per $\mathrm{Cu}$ estimated from the acoustic resonance alone in previous work ${ }^{4}$. Even if we consider that our estimation may be too large by $30 \%$, the change in the exchange energy is still much larger than the $U_{0} \cong 25 \mathrm{~J} \mathrm{~mol}^{-1} \simeq 0.26 \mathrm{meV} \mathrm{f.u} .^{-1}=3 \mathrm{Kf} . \mathrm{u}^{-1}=$ $1.5 \mathrm{~K}$ per planar Cu condensation energy ${ }^{15,16}$.

Our results reveal two important conclusions for spin excitations of optimally doped YBCO. First, the optical resonance reported earlier ${ }^{24,25}$ is actually incommensurate and this naturally explains the large $Q$ widths previously reported. Second, our determination of the dynamical susceptibility in absolute units enables an estimation of the change of the magnetic excitation energy available to the superconducting condensation energy $y^{4,14,26}$. We find that the magnetic exchange energy is about 15 times larger than that of the superconducting condensation energy, thus indicating that magnetism can be the driving force for electron pairing and superconductivity.

Received 25 January 2006; accepted 31 July 2006; published 27 August 2006.

References

1. Scalapino, D. J. The case for $d_{x^{2}-y^{2}}$ pairing in the cuprate superconductors. Phys. Rep. 250, 330-365 (1995).

2. Chubukov, A., Pines, D. \& Schmalian, J. in The Physics of Superconductors, Vol I, Conventional and High- $T_{c}$ Superconductors (eds Bennemann, K. H. \& Ketterson, J. B.) 495-590 (Springer, Berlin, 2003).

3. Scalapino, D. J. \& White, S. R. Superconducting condensation energy and an antiferromagnetic exchange-based pairing mechanism. Phys. Rev. B 58, 8222-8224 (1998).

4. Demler, E. \& Zhang, S.-C. Quantitative test of a microscopic mechanism of high-temperature superconductivity. Nature 396, 733-735 (1998).

5. Maier, Th. A. On the nature of pairing in the two-dimensional $t-J$ model. Physica B 359-361, 512-514 (2005).

6. Rossat-Mignod, J. et al. Neutron scattering study of the $\mathrm{YBa}_{2} \mathrm{Cu}_{3} \mathrm{O}_{6+x}$ system. Physica C 185, 86-92 (1991).

7. Mook, H. A. et al. Polarized neutron determination of the magnetic excitations in $\mathrm{YBa}_{2} \mathrm{Cu}_{3} \mathrm{O}_{7}$. Phys. Rev. Lett. 70, 3490-3493 (1993).

8. Mook, H. A. et al. Spin fluctuations in $\mathrm{YBa}_{2} \mathrm{Cu}_{3} \mathrm{O}_{6.6}$. Nature 395, 580-582 (1998).

9. Fong, H. F. et al. Spin susceptibility in underdoped $\mathrm{YBa}_{2} \mathrm{Cu}_{3} \mathrm{O}_{6+x}$. Phys. Rev. B 61, 14773-14786 (2000).

10. Dai, P., Mook, H. A., Hunt, R. D. \& Doğan, F. Evolution of the resonance and incommensurate spin fluctuations in superconducting $\mathrm{YBa}_{2} \mathrm{Cu}_{3} \mathrm{O}_{6+x}$. Phys. Rev. B 63, 054525 (2001). 
11. Stock, C. et al. From incommensurate to dispersive spin-fluctuations: The high-energy inelastic spectrum in superconducting $\mathrm{YBa}_{2} \mathrm{Cu}_{3} \mathrm{O}_{6.5}$. Phys. Rev. B 71, 024522 (2005).

12. Hayden, S. M., Mook, H. A., Dai, P., Perring, T. G. \& Doğan, F. The structure of the high-energy spin excitations in a high-transition-temperature superconductor. Nature 429, 531-534 (2004).

13. Dai, P. et al. The magnetic excitation spectrum and thermodynamics of high- $T_{c}$ superconductors. Science 284, 1344-1347 (1999).

14. Kee, H-Y., Kivelson, S. A. \& Aeppli, G. Spin-1 neutron resonance peak cannot account for electronic anomalies in the cuprate superconductors. Phys. Rev. Lett. 88, 257002 (2002).

15. Loram, J. W., Mirza, K. A., Cooper, J. R. \& Tallon, J. L. Specific heat evidence on the normal state pseudogap. J. Phys. Chem. Solids 59, 2091-2094 (1998).

16. Lortz, R. et al. Evolution of the specific-heat anomaly of the high-temperature superconductor in $\mathrm{YBa}_{2} \mathrm{Cu}_{3} \mathrm{O}_{7}$ under the influence of doping through application of pressure up to $10 \mathrm{GPa}$. J. Phys. Condens. Matter 17, 4135-4145 (2005).

17. Woo, H. et al. Mapping spin-wave dispersions in stripe-ordered $\mathrm{La}_{2 x} \mathrm{Sr}_{x} \mathrm{NiO}_{4}(x=0.275,0.333)$. Phys. Rev. B 72, 064437 (2005).

18. Reznik, D. et al. Dispersion of magnetic excitations in optimally doped superconducting $\mathrm{YBa}_{2} \mathrm{Cu}_{3} \mathrm{O}_{6.95}$. Phys. Rev. Lett. 93, 207003 (2004).

19. Regnault, L. P. et al. Spin dynamics in the high- $T_{c}$ superconducting system $\mathrm{YBa}_{2} \mathrm{Cu}_{3} \mathrm{O}_{6+x}$. Physica $B$ 213/214, 48-53 (1995).

20. Chen, W. Q. \& Weng, Z. Y. Spin dynamics in a doped-Mott-insulator superconductor. Phys. Rev. B 71, $134516(2005)$.

21. Hayden, S. M. et al. High-frequency spin waves in $\mathrm{YBa}_{2} \mathrm{Cu}_{3} \mathrm{O}_{6.15}$. Phys. Rev. B 54, R6905-R6908 (1996).

22. Reznik, D. et al. Direct observation of optical magnons in $\mathrm{YBa}_{2} \mathrm{Cu}_{3} \mathrm{O}_{6.2}$. Phys. Rev. B 53, R14741-R14744 (1996).
23. Dahm, T. et al. Nodal quasiparticle lifetimes in cuprate superconductors. Phys. Rev. B 72, 214512 (2005).

24. Pailhes, S. et al. Resonant magnetic excitations at high energy in superconducting $\mathrm{YBa}_{2} \mathrm{Cu}_{3} \mathrm{O}_{6.85}$. Phys. Rev. Lett. 93, 167001 (2004).

25. Pailhes, S. et al. Two resonant magnetic modes in an overdoped high $T_{c}$ superconductor. Phys. Rev. Lett. 91, 237002 (2003).

26. Chakravarty, S. \& Kee, H-Y. Measuring condensate fraction in superconductors. Phys. Rev. B 61, 14821-14824 (2000).

\section{Acknowledgements}

We thank E. Dagotto, Z. Y. Wen and F. C. Zhang for helpful discussions. This work is supported by the US DOE Office of Science, Division of Materials Science, Basic Energy Sciences under contract No. DE-FG02-05ER46202 (H.W. and P.D.). Oak Ridge National Laboratory is supported by the US DOE under contract No. DE-AC05-00OR22725 with UT/Battelle LLC. S.M.H. is supported by the UK

EPSRC. D.J.S. would like to acknowledge the Center for Nanophase Material Science at Oak Ridge National Laboratory for their support.

Correspondence and requests for materials should be addressed to P.D. or H.A.M.

Supplementary Information accompanies this paper on www.nature.com/naturephysics.

\section{Competing financial interests}

The authors declare that they have no competing financial interests.

Reprints and permission information is available online at http://npg.nature.com/reprintsandpermissions/ 